\title{
Whethering Station: thin surface, thick space
}

In Surface Architecture (2002) David Leatherbarrow and Mohsen Mostafavi dissect Gottfried Semper's well established emphasis on the importance of surface for architecture:

the surface of buildings [is] the subject matter of architectural design. The autonomy of the surface presumes a distinction between the structural and nonstructural elements of the building, between frame and cladding ... the relationship between structure and skin has preoccupied much architectural production ... and remains contested today. The site of this contest is the architectural surface (Leatherbarrow and Mostafavi, 2002: 7).

This project continues Leatherbarrow and Mostafavi's "contest" through the design of a modest pavilion titled Whethering Station. ${ }^{1}$ The design was developed using now ubiquitous parametric modelling software in preparation for a novel fabrication process using double curved composite materials developed at The University of Queensland's (UQ) Centre for Advanced Materials Processing and Manufacturing. Whilst technically experimental, the work is underpinned by a critical position that surface is "the" primary instrument in the conception and evolving of built form.

The design began with an idea-an analogue process, the scribbling of diagrams to explore structural and spatial ideas to deploy thin $(4-6 \mathrm{~mm})$ bio-composite materials without framing. A simple line drawing suggested a double curved surface with buttressing for rigidity and load distribution akin to the works of Frei Otto, Felix Candela et al. Andrew Benjamin (2006) describes the line's role in "projecting" surface as "that which distributes volume ... the line becomes the architectural correlate to the surface" (14). Whether analogue or digital, the line predicts surface; a condition which defines the object in context and the delineation of volumes within.

The line here depicts an idealized form, simultaneously conceptual and technical. Benjamin suggests that Semper's design process was the opposite: for Semper "the wall [i.e. the frame and its surface] is that which brings about spatial enclosure" (Benjamin, 2006: 21). The line's significance in relation to surface and space is particularly relevant in the sectional drawing in both the abstract sketch and traditional technical documents. ${ }^{2}$ Design intent is thus understood as both 
the lines that define the external extremities of cladding, which in turn establishes form, pattern etc. and internally, those that describe linings which demarcate programmatic subdivisions and the extent of space-the experience of architecture's interior.

Semper's "frame" is embedded between these lines, at times wrapped tight by these membranes as if vacuum packed for economy. In other instances, they bulge and billow to meet the line of intent. Myriam Blias "wonders about the possibility of an architecture of cladding independent of its structural frame, with regard to architecture's representative role" (1996: 1). She suggests that the detail contained in the inner workings of the section remains mute, only ever being seen in the context of 2D drawings, but adds that "the organization of space, the configuration of a building's envelope and the treatment of its cladding constitute a most important part of what is given to architects to reflect upon" (1). Conceptually, the Whethering Station obliterates the mute workings of the section and explores the possibility of simply building the surface implied by a $6 \mathrm{~mm}$ "thin" line.

Fig. 1 John de Manincor (2017). [Sectional Concept Sketch]

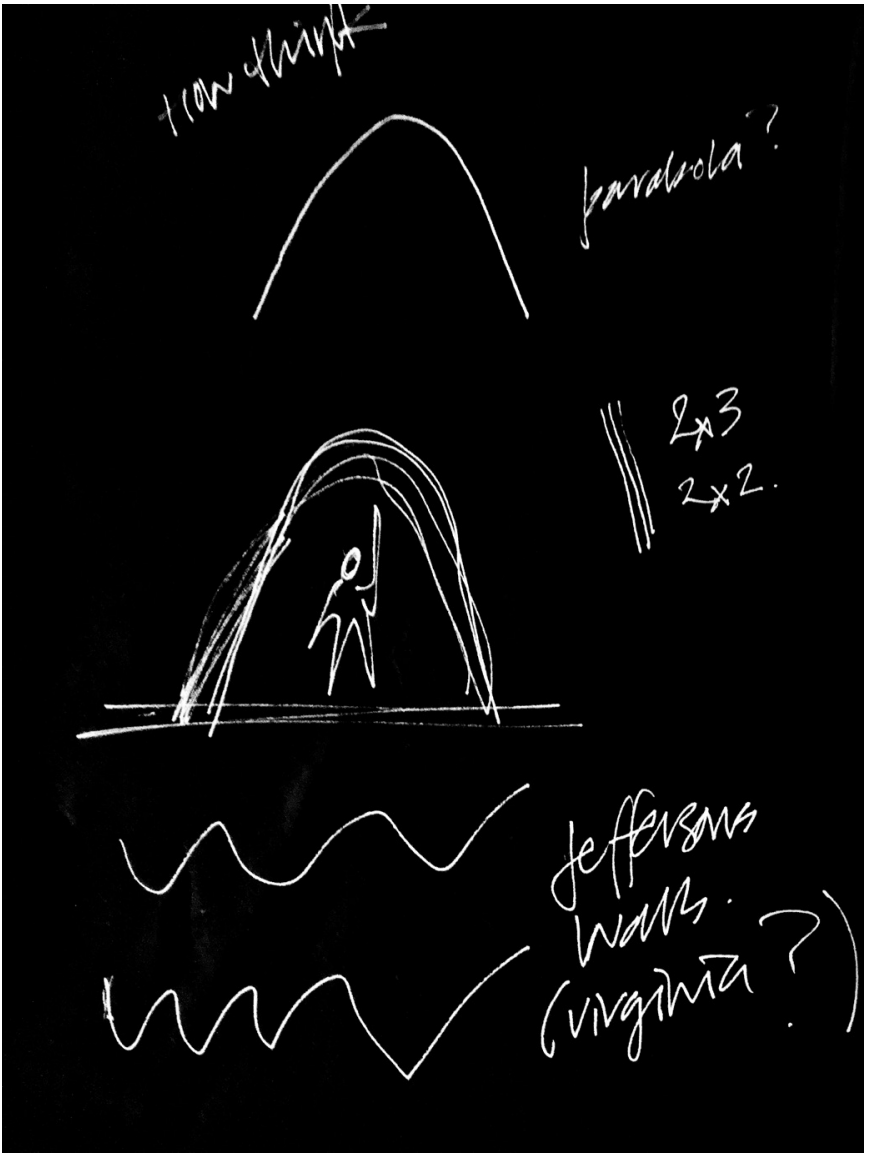

From the initial scribble, the project was developed in the digital environment. Working with NURBS and surface modelling tools such as Rhino or Maya highlights the abstract nature of surface as a concept by developing ideas about form and space which, in the virtual world, essentially have zero thickness. Like the outer lines of the 2D section drawing, the distance between surfaces in these environments can literally follow pragmatic constraints of structure or take on more expressive forms. Seyed Islami suggests that "the majority of today's modelling 
software is surface-driven, pushing the architects towards designs which exploit the thinness and complexity of digital surfaces" (2007: 645). ${ }^{3}$ The Whethering Station is not the result of a specific interest the "inherent 'vitalism' of computer-generated series" (Vidler, 2000: 227) nor "parametricism" (Schumacher, 2009), rather it explores the possibility of spatial definition through a surface without apparent structure. Design through surface modelling of zero or minimal thickness also brings forth parallels with American philosopher Avrum Stoll's "conceptual problem about what counts as a surface"-for instance, what constitutes the surface of a lake (the water or the land below?), or, in the case of Leonardo da Vinci's observations on the surface between oil and water (1992:196). These natural surfaces without thickness directly correlate to the way in which surface is deployed in the digital environment (196). That is to say, while the representation of surface in virtual space is a conceptual proposition-rather than being an immediate material state-this proposition has direct parallels to physical perceptions of space. Thus, the hypothesis here is that surface is that condition where materials end and where space, or form, begin. In that sense the surface of the Whethering Station, in its completed form, will be the outer molecules of the proposed dichroic paint finish.

Therefore, this experimental structure simultaneously explores thinness-thinness as spatial and technical propositions. The project was developed in parallel with a small renovation to the School of Architecture at UQ designed by m3architecture. The architect's idea was to draw on the inherent qualities of a mural in the space painted by Pancho Guedes [circa 1977] "which tells a story of chance meetings, shared ideas and joy” (m3architecture, 2016).

Fig. 2 Mural by Pancho Guedes (1977) Photo: () Brett Boardman]

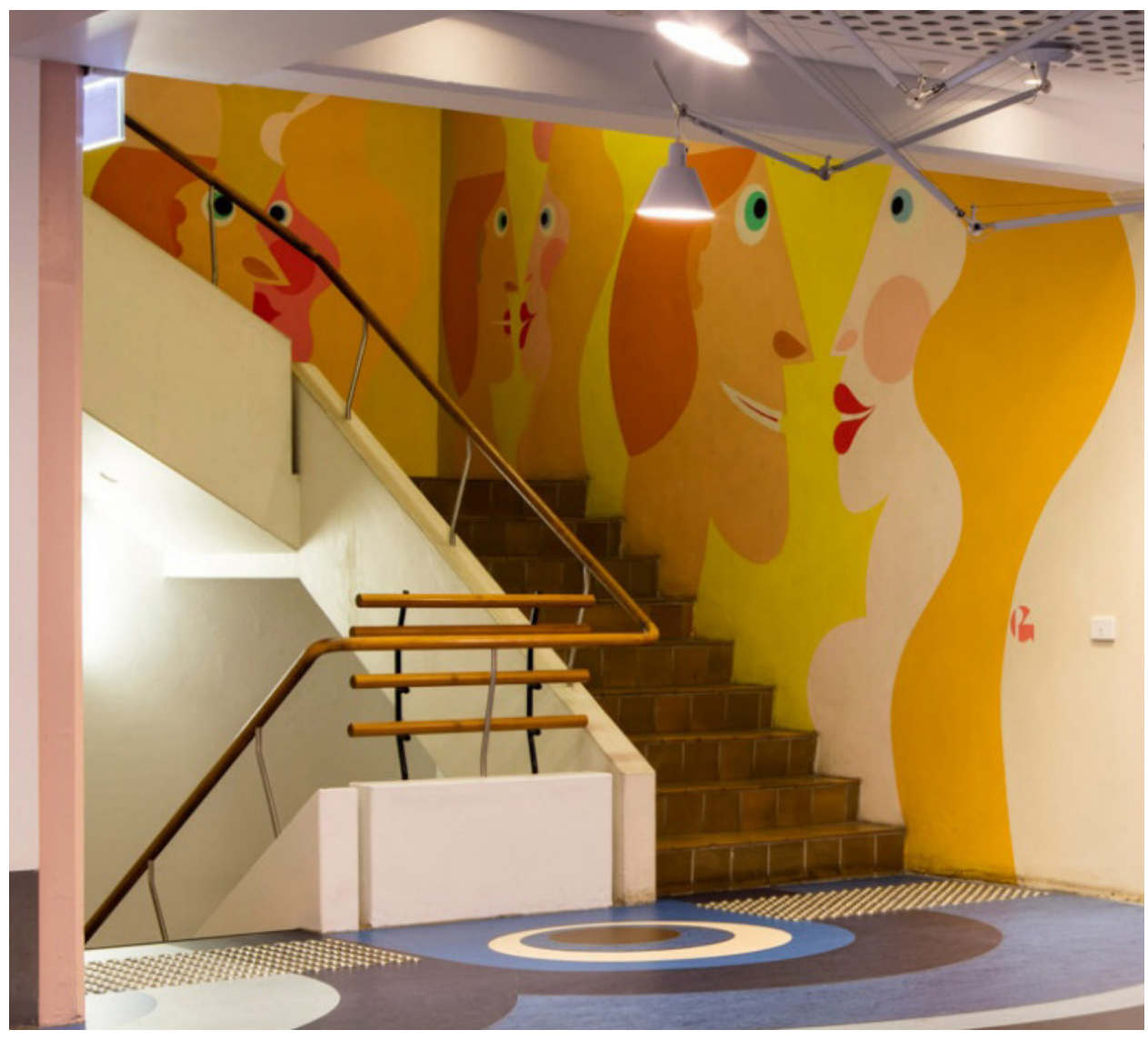


Taking clues from this observation, the pavilion abstracts the faces of the Guedes mural in plan, whilst in section, the double curved enclosure notionally represents the flowing hair of the protagonists matted together to create a sheltered seating space for casual conversation-a place to ask questions "whether" someone else is present or not. One reading of the work is that its billowing form is comparable to Semper's textile analogies. However, the research attempts to invert Semper and even Adolf Loos' ideas of wrapping over a "core-form" (Hartoonian, 2004: 47), so the pavilion has no frame, with cladding and structure melding to define enclosure. Monica Ponce de Leon and Nader Tehrani expressed a desire to redefine tectonics as both "the apparent and finished membranes of construction, and, importantly, their subsequent effects" (Ponce de Leon \& Tehrani, 2002: 24). Here these membranes are tightly compressed; the tectonic is equally the formal profile and the implied mass of its interior.

Fig. 3 Design Evolution [Design: John
de Manincor. Image: Shuwei Zhang]

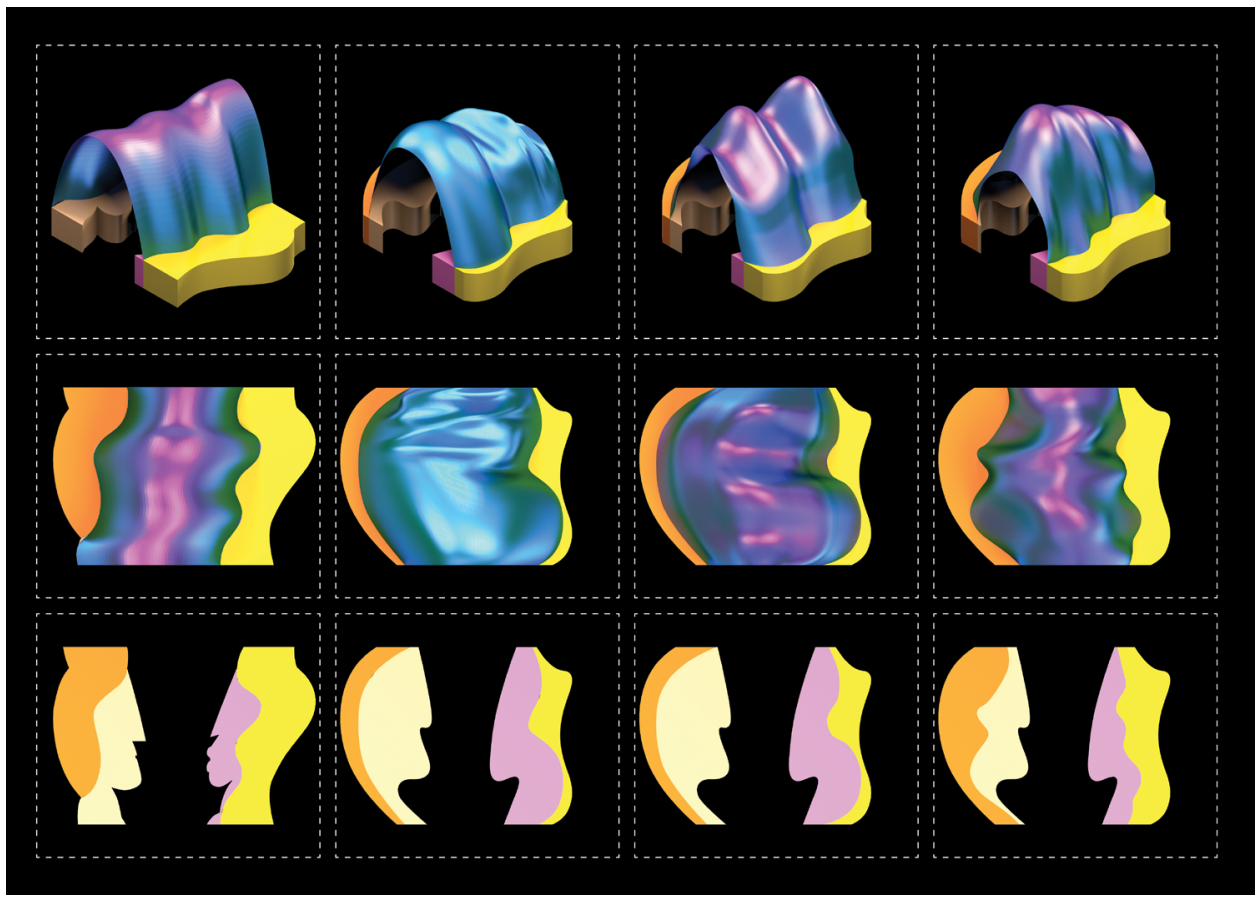

Beyond metaphoric and spatial aspirations, this project deploys a unique fabrication method. The shell will be formed of two layers of $3 \mathrm{~mm}$ thick bio-composite sheets comprising bio-resins reinforced with woven hemp matting. Each layer is arranged in an offset grid of panels up to $600 \mathrm{~mm}$ square. Panels will be laminated together with the complex curvature forming a self-supporting structure stiffened by the undulating buttressing of the form in a manner akin to Eladio Dieste's Church at Atlantida in Uruguay (1952). To fabricate the double curved composite materials would normally require formwork or moulds to establish and maintain desired geometry during the curing process. The constantly shifting geometry of the surface would thus need more than 150 bespoke moulds. It would be feasible to create such moulds by milling expanded polystyrene with a CNC machine or robotic arm, or by forming plywood diaphragms. However, as the project is a one-off the moulds would be disposed of potentially as landfill or at best-in the case of polystyrene-recycled. Termed the Pixel Table, the system was developed with UQ's Composites Group. Taking clues from a child's nail impression toy, each rod on the table can be vertically adjusted to the 
geometry determined in the design model using a robotic arm. Analyses in the digital model identify where the design curvature exceeds the minimum radius that the pixels can form and the design model adjusted accordingly. Each panel is vacuum-formed on the Pixel Table then trimmed using a water-jet cutter. The butt-joints between panels will be taped and set then finished with heat sensitive dichroic paint.

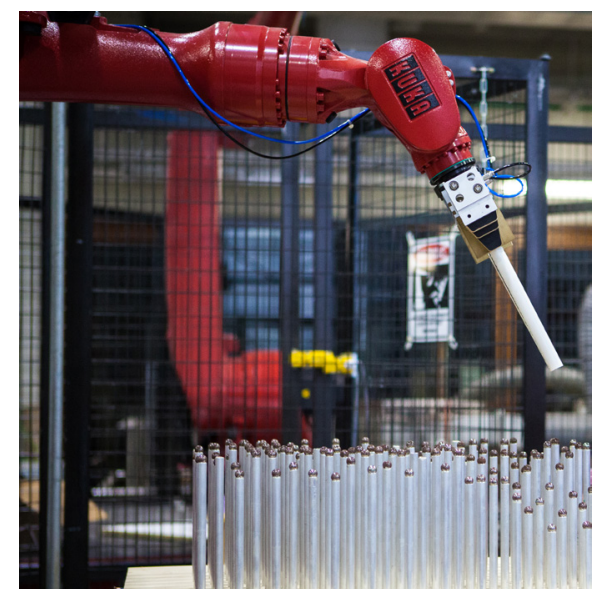

Fig. 4 "Pixel Table" and robotic arm [Photo: Shuwei Zhang]

Fig. 5 Biocomposite prototypes [Photo: John Milne]

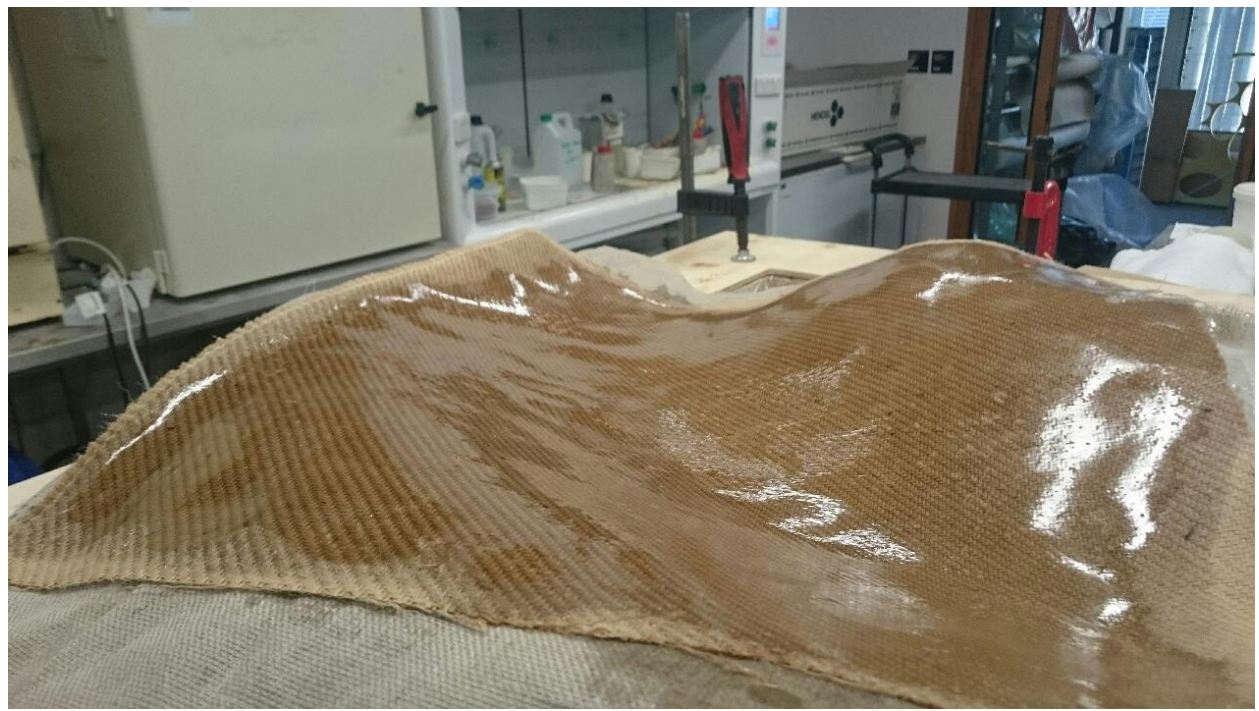

Questions of "why surface?" are well documented in the writing of Avrum Stroll. In his essay Reflections on Surfaces (1992), he refers to J.J. Gibson's ideas of perception through surface:

Why, in the triad of medium, substances, and surfaces, are surfaces so important? The surface is where most of the action is. The surface is where light is reflected or absorbed, not the interior of the substance ... the surface is where vibrations of the substances are transmitted into the medium. (192)

Hence, there is little or no interior substance to the material that defines the Whethering Station. It is the curious geometry of the surfaces and the space they define that transmits the "action"; that "action" is, of course, open to interpretation by those who engage with it. For Nader Tehrani and Monica Ponce de Leon of Office $d A$, the alibis for the arbitrary in their work is critical. As they note, 
"one's alibi may be false, a mere pretext to get away with the crime" (Ponce de Leon \& Tehrani, 2002: 22). It is acknowledged that the selection of the metaphors derived from the aforementioned Pancho Guedes mural outlined by m3architecture is a somewhat arbitrary means to generate "action". It is simply a starting point, the alibi to generate and test conceptual ideas concerning surfaces, and in turn, to develop new modes of fabrication.

Fig. 6 Wethering Station (2018) [Design: John de Manincor. Image: Oliver Shearer]

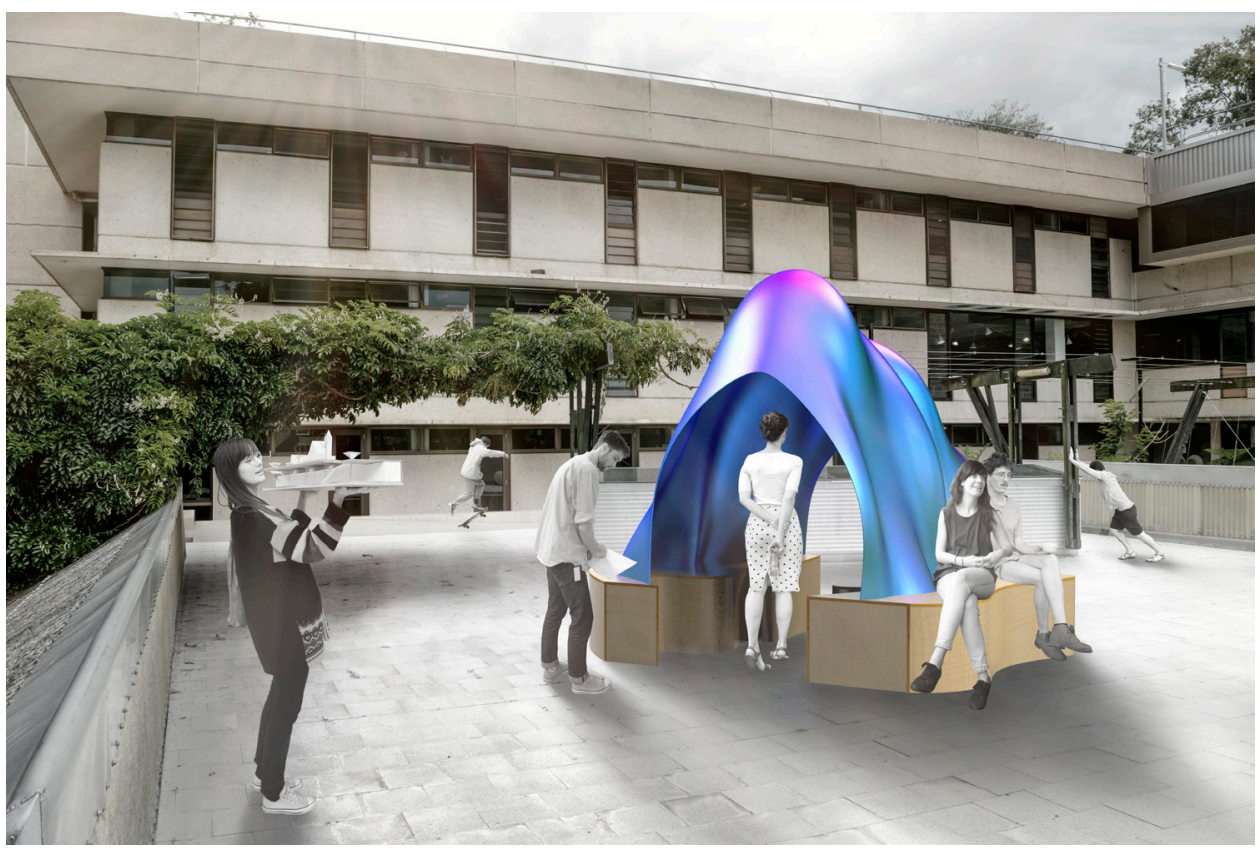


Endnotes

1 NOTE: The spelling of Whethering here is intentional, this modest project creates spaces where conversations might occur, where questions might be asked, whether or not these questions can be answered is entirely up to the users.

2 The term "traditional" here intends to be provocative, as construction documentation is undergoing radical change with the proliferation technologies like 3D pdf files and file-to-factory processes.

3 Based on this observation it would be reasonable to argue Islami is referring to NURBS or surface modelling packages such as Rhino, Maya or AutoCAD, rather than the solid forms of say a Revit model.
Benjamin A. (2006). Surface Effects: Borromini, Semper, Loos. The Journal of Architecture, No. 11(1), 1-35.

Blais, M. (1996). Cladding and representation: between scenography and tectonic. In Proceedings of Construction of tectonics for the Post-Industrial World-ACSA European Conference, Copenhagen 2009. 125-129.

Gibson, J. The Ecological Approach to Visual Perception (Boston, MA: Houghton-Mif- flin 1979), in Stroll, A. Reflections on Surfaces, Canadian Journal of Philosophy, Vol. 22, No. 2 (June, 1992), 191-210.

Hartoonian, G. (2004). Surface: A-Tectonic of Roofing and Wrapping, Architectural Theory Review, 9:1, 45-58.

Islami, S.Y. (2007) Surfacedriven Architecture-Moving Beyond the Ornament/Structure Opposition. In Proceedings of Em'body'ing Virtual Architecture: The Third International Conference of the Arab Society for Computer Aided Architectural Design (2007), 672-675.

Leatherbarrow, D, and Mohsen M. (2002). Surface Architecture. Cambridge, MA: MIT Press.

m3architecture. (2016). University of Queensland Architecture School. Retrieved from http:// www.m3architecture.com/ projects/university-queenslandschool-architecture/

Ponce de Leon, $\mathrm{M}$ and Tehrani, N. (2002). Versioning: Connubial Reciprocities of Surface and Space. Architectural Design, 72 , (5) September/October, 18-28.

Schumacher, P. (2009).

Parametric Patterns. Architectural Design-Patterns of Architecture. 79, (6), 28-41.

Stroll, A. (1992). Reflections on Surfaces. Canadian Journal of Philosophy, 22, (2), 191-210.

Vidler, A. (2000) Warped Space Art. Architecture, and Anxiety in Modern Culture. Cambridge, MA: MIT Press. 